\title{
Croup, tratamiento actual
}

\section{Croup management}

\section{Dra. Claudia Fuentes ${ }^{1}$, Dr. Rubén Peña ${ }^{2}$, Michelle Vinet ${ }^{3}$, Dr. Daniel Zenteno ${ }^{1}$}

1 Pediatra Especialista en Enfermedades Respiratorias. Profesor Asistente, Departamento de Pediatría, Facultad de Medicina, Universidad de Concepción. Hospital Guillermo Grant Benavente.

${ }^{2}$ Becado Pediatría, Facultad de Medicina, Universidad de Concepción. Hospital Guillermo Grant Benavente.

${ }^{3}$ Interna de Medicina, Facultad de Medicina, Universidad de Concepción.

\section{CROUP MANAGEMENT}

Croup is a frequent cause of outpatient and emergency consultation. It is frequently triggered by a viral respiratory infection and characterized by an abrupt onset. We describe current studies about the treatment of croup. The use of systemic corticosteroids stands out as choice treatment, and nebulized epinephrine as short term therapy while waiting for the anti-inflammatory effect of steroids.

Key words: Croup, laryngitis, stridor, corticosteroids.

\section{RESUMEN}

El Croup (laringitis aguda) es motivo frecuente de consulta en servicios de urgencia y policlínicos pediátricos. Generalmente es gatillado por una infección respiratoria viral y se caracteriza por un inicio abrupto. En el presente texto se describen los estudios vigentes acerca de su tratamiento. Destacan los corticoides sistémicos como terapia de elección y la adrenalina nebulizada como terapia de acción corta en espera de la acción antinflamatoria esteroidal.

Palabras clave: Croup, laringitis, estridor, corticosteroides.

\section{INTRODUCCIÓN}

El Croup, Ilamado también laringitis aguda, es una infección respiratoria habitualmente viral que se caracteriza por estridor, tos "perruna" y disfonía. Se presenta en brotes de acuerdo al virus que sea epidemiológicamente predominante (1).

Ocurre principalmente en los meses de otoño e invierno, siendo la edad más frecuente entre los 6 meses y los 5 años, con un peak de incidencia a los 2 años $(1,2)$. Se presenta más en hombres que en mujeres con una relación 1-4:1 (3). El 3\% de los niños tendrá un episodio de croup antes de los 6 años de vida (1). Con respecto a la severidad, un estudio realizado en Canadá demostró que el 85\% correspondió a Croup leve y menos del 5\% requirió hospitalización (4).

El agente etiológico más frecuente es el virus Parainfluenza tipos 1 y 3 (5). Otros virus implicados son Influenza A, Influenza $\mathrm{B}$, Adenovirus y Virus respiratorio sincicial (6). Existen además estudios que han demostrado que en pacientes pediátricos con Croup hay una asociación con metapneumovirus y coronavirus $(7,8)$.

\section{Correspondencia:}

Dra. Claudia Fuentes Sáez

Departamento Pediatría

Facultad de Medicina

Universidad de Concepción

Chacabuco esquina Janequeo s/n

Concepción, Chile

Teléfonos: (41) 2204852 - (41) 2204877

e-mail: claufuentessaez@gmail.com

\section{FISIOPATOLOGÍA Y CLÍNICA}

La infección viral produce una inflamación generalizada de la vía aérea, originando edema e inflamación de la mucosa epitelial. La región subglótica se ve especialmente afectada, con disminución de su diámetro (9). En esta zona se produce una mayor resistencia al paso del flujo aéreo en inspiración lo que provoca un flujo turbulento y genera el signo cardinal que es el estridor. Si la inflamación aumenta, el paciente presentará signos de apremio respiratorio tales como tiraje, asincronía de los movimientos toraco-abdominales, fatiga y progresivamente falla respiratoria (10).

Por lo general luego de 1-3 días de síntomas de resfrío aparecen en forma abrupta y durante la noche dificultad respiratoria, estridor, tos perruna y disfonía; generalmente sin fiebre, ni aspecto tóxico. Los casos moderados de croup pueden tener taquipnea, aleteo nasal, retracción supraesternal e intercostal. En los casos más severos se agrega cianosis y disminución del murmullo pulmonar (1). El $60 \%$ de los pacientes presentará resolución clínica de su cuadro dentro de 48 horas, aunque algunos de ellos podrían presentar síntomas hasta una semana (11).

\section{DIAGNÓSTICO}

El diagnóstico es clínico. Los exámenes de laboratorio no son recomendados en forma rutinaria. Se debe conocer la evolución natural de la enfermedad porque hay pacientes que no presentan el Croup "clásico", lo que puede llegar a generar un diagnóstico erróneo. Así también tener en consideración diagnósticos diferenciales.

ISSN 0718-333X. Derechos reservados. 
Tabla №1. Diagnóstico diferencial de croup.

\begin{tabular}{|c|}
\hline Epiglotitis \\
\hline Croup Espasmódico \\
\hline Traqueitis bacteriana \\
\hline Edema Angioneurótico \\
\hline Cuerpo extraño en la vía aérea \\
\hline Trauma Laríngeo \\
\hline Quemadura en la vía aérea \\
\hline Amigdalitis severa \\
\hline Absceso retrofaríngeo o parafaríngeo \\
\hline Difteria \\
\hline
\end{tabular}

Un paciente con Croup que no evoluciona de manera habitual o tiene características que hacen sospechar otro diagnóstico requiere evaluación endoscópica de la vía aérea, considerando principalmente la presencia de un cuerpo extraño como diagnóstico diferencial (1).

Para evaluar severidad se proponen la escala de Downes (Tabla 2) que es la más conocida en nuestro medio y el puntaje de

Tabla ํ⒉ Escala de Downes.

\begin{tabular}{|c|c|}
\hline Grado & Clínica \\
\hline I & $\begin{array}{r}\text { Estridor al Ilanto 0 actividad } \\
\text { Ausencia de retracción }\end{array}$ \\
\hline II & $\begin{array}{c}\text { Estridor inspiratorio en reposo } \\
\text { Retracciones supraesternal o intercostal en reposo } \\
\text { Sin agitación }\end{array}$ \\
\hline III & $\begin{array}{r}\text { Gran estridor inspiratorio o bifásica } \\
\text { Signos de dificultad respiratoria }\end{array}$ \\
\hline IV & $\begin{array}{c}\text { Falla respiratoria inminente: tos débil, presencia de } \\
\text { alteración en nivel de consciencia, signos de hipoxemia }\end{array}$ \\
\hline
\end{tabular}

Tabla No3. Escala de Wesley.

\begin{tabular}{|c|c|}
\hline & Estridor \\
\hline 0 & Ausente \\
2 & En reposo, audible con estetoscopio \\
2 & En reposo, audible sin estetoscopio \\
\hline & Retracción \\
\hline 0 & Ausente \\
1 & Retracción leve \\
2 & Retracción moderada \\
3 & Retracción severa \\
\hline & Entrada de aire \\
\hline 0 & Normal \\
1 & Disminuida pero audible \\
2 & Muy disminuida, poco audible \\
\hline & Cianosis \\
\hline 0 & Ausente \\
4 & Con la agitación \\
5 & En reposo \\
\hline
\end{tabular}

Puntaje:

0-1 Croup leve / 2-7 Croup moderado / $\geq 8$ Croup severo
Westley (Tabla 3), siendo la primera de mayor utilidad clínica y la segunda para estudios de investigación.

\section{TRATAMIENTO}

- Cuidados generales y aporte de oxígeno

Se debe mantener al paciente en un ambiente tranquilo, en posición cómoda. Sentar al niño en brazos de los padres es una buena forma de disminuir su agitación (12). Existe consenso que indica que el oxígeno puede ser beneficioso; se recomienda aplicar a centímetros de la nariz y boca (12). El aire humidificado no ha mostrado ser efectivo (13). El Heliox, mezcla gaseosa respirable de helio y oxígeno disminuye el flujo turbulento y facilita la entrada de oxígeno; no existe aún evidencia suficiente para establecer su efecto como terapia de rutina en los casos de Croup (14).

\section{- Corticoides}

\section{Corticoides vs placebo}

Existen 3 meta-análisis al respecto, siendo el más importante el que incluye 3700 pacientes, a quienes se le administró corticoides vía oral, intramuscular o nebulizado vs placebo (15). Los resultados mostraron que con el uso de corticoides hubo una mejoría del score clínico, reducción de la estadía en unidad de emergencia, reducción en la recurrencia y reducción en el uso de adrenalina (15).

De los estudios randomizados controlados que evalúan corticoides vs placebo el mayor incluyó 720 pacientes con Croup leve. Se evaluó el uso de dexametasona $0,6 \mathrm{mg} / \mathrm{kg}$ oral vs placebo. Muestra una menor reconsulta dentro de los siguientes 7 días en los pacientes que habían recibido dexametasona $(7,3 \%)$ vs $15,3 \%$ en los que recibieron placebo, $p<0,001$ (16).

\section{Comparación vía de administración de corticoides}

Nebulización vs intramuscular

Al comparar el uso de budesonida nebulizada vs dexametasona por vía intramuscular no hubo diferencias en puntaje de severidad, en ambos grupos se observó mejoría (17).

\section{Vía intramuscular vs oral}

Comparando dexametasona intramuscular vs betametasona oral, el estudio más reciente es del año 2006 con 52 pacientes. Su outcome primario fue el puntaje clínico según escala de Westley a las 4 horas sin observarse diferencias significativas entre ambos grupos (18). Como recomendación se prefiere la administración oral, a menos que exista intolerancia por esta vía o el paciente se encuentre en un estado de gravedad (19). 


\section{Prednisolona v/s Dexametasona}

En 113 pacientes con Croup leve a moderado, de edades entre 3 a 142 meses, se comparó la frecuencia de reconsulta entre 2 grupos: la mitad recibió prednisolona $2 \mathrm{mg} / \mathrm{k}$ oral y el resto dexametasona $0,15 \mathrm{mg} / \mathrm{k}$ oral. El primer grupo volvió a consultar en un $29 \%$, el segundo solo un $7 \%$ (20). Los autores comentan que prednisolona y dexametasona parecieran ser igual de eficaces cuando son administradas por primera vez a un paciente con Croup, pero la reconsulta en un servicio asistencial es más frecuente en los pacientes que recibieron prednisolona. Esto podría explicarse por la menor vida media de esta última (20). En nuestro medio la prednisona es el corticoide oral más ampliamente utilizado ( $1 \mathrm{mg} / \mathrm{kg} /$ día por 2-3 días), sin embargo no existen investigaciones científicas que sustenten su eficacia.

\section{Dosis de corticoides}

No existen estudios que comparen una dosis versus múltiples dosis. Se sabe que el efecto antinflamatorio de la dexametasona se mantiene por dos a cuatro días por lo que parece innecesario administrar dosis suplementarias, debido a que el cuadro clínico por lo general se resuelve en 72 horas (20). Existen estudios que evalúan distintas dosis de este medicamento, sin encontrar diferencias significativas entre ellas (20). La dosis convencional de dexametasona es de 0,6 mg/kg, sin embargo hay estudios que sugieren que $0,15 \mathrm{mg} / \mathrm{kg}$ también sería efectivo. Dosis más altas podrían presentar mayor beneficio en Croup de mayor severidad (21).

El uso de corticoides está asociado a menor uso de adrenalina, menos re-consultas y menor tiempo de estadía hospitalaria. La dosis óptima aún no está definida (22).

\section{Adrenalina}

Los efectos de la adrenalina se observan a los 10 minutos, perdurando hasta dos horas. Se recomienda no utilizarla por horario sino según respuesta clínica. Los efectos adversos más frecuentes son taquicardia y palidez (23). En el Croup severo disminuye la necesidad de intubación y traqueostomía (24). Se encuentran estudios que analizan el efecto de la adrenalina común o racémica, observándose mejoría del puntaje clínico a los 30 minutos $(19,25,26)$. Ambos tipos de adrenalina son igualmente efectivas. Una dosis de $0,5 \mathrm{ml}$ de adrenalina racémica al 2,25\% equivale a $5 \mathrm{ml}$ de adrenalina común en dilución 1/1000.

\section{Otras terapias}

Con respecto al uso de analgésicos, antipiréticos, antitusivos, descongestionantes, broncodilatadores $\beta 2$ de acción corta no existen estudios que demuestren efectividad (19). El uso de antiinflamatorios no esteroidales no está avalado en la literatura en laringitis obstructiva (19).

\section{CONCLUSIONES}

Luego de 50 años de controversia en el manejo del Croup, Ios corticoides se han establecido como el tratamiento de elección para su tratamiento. La adrenalina es útil como terapia de acción rápida, mientras se espera la acción de esteroides. La asociación de ambos fármacos ha mejorado los resultados del tratamiento, evitando hospitalizaciones.

\section{REFERENCIAS}

1. Holmgren N, Caussade S. Obstrucción de vía aérea superior. En: Sánchez I, Prado F. Enfoque Clínico de las Enfermedades Respiratorias del Niño. Ediciones Universidad Católica; Santiago de Chile, 2007, pág $147-164$

2. Everard M. Acute Bronchiolitis and Croup. Pediatr Clin N Am 2009;56:119-133

3. Denny F, Murphy T, Clyde W, Collier A, Henderson F. Croup: a 11-year study in pediatric practice. Pediatr 1983; 71: 871-76

4. Johnson D, Williamson J. Health care utilization by children with croup in Alberta. Pediatr Res 2003; 53: 185A

5. Marx A, Torok T, Holman R, Clarke M, Anderson L. Pediatric hospitalizations for croup (laryngotracheobronchitis): biennial increases associated with human parainfluenza virus 1 epidemics. J Infect Dis 1997; 176: 1423-27

6. Chapman R, Henderson F, Clyde W, Collier A, Denny F. The epidemiology of tracheobronchitis in pediatric practice. Am J Epidemiol 1981; 114: 786-97

7. Williams J, Harris P, Tollefson SJ, Halburnt-Rush L et al. Human Metapneumovirus and Lower Respiratory Tract Disease in Otherwise Healthy Infants and Children. N Engl J Med 2004;350:443-50

8. Van der Hoek L, Sure K, Ihorst G, Stang A et al. Croup is associated with the novel coronavirus NL63. Adv Exp Med Biol 2006; 581: 485-91 9. Cherry J. Croup (laryngitis, laryngotracheitis, spasmodic croup, laryngotracheobronchitis, bacterial tracheitis, and laryngotracheobronchopneumonitis). In: Feigin R. Textbook of Pediatric Infectious Diseases, 5th Ed. Philadelphia, Elsevier, 2004: 252-65

10. Davis G, Cooper D, Mitchell I. The measurement of thoracoabdominal asynchrony in infants with severe laryngotracheobronchitis. Chest 1993; 103: 1842-48

11. Johnson D, Williamson J. Croup: duration of symptoms and impact on family functioning. Pediatr Research 2001; 49: 83A

12. Johnson D, Klassen T, Kellner J. Diagnosis and management of croup: Alberta Medical Association clinical practice guidelines. Alberta: Alberta Medical Association, 2005

13. Moore M, Little P. Humidified air inhalation for treating croup. Cochrane Database Syst Rev 2006; 3: CD002870

14. Vorwerk C, Coats T. Heliox for croup in children (Review). Cochrane Database Syst Rev. 2012;10:CD006822.

15. Russell K, Wiebe N, Saenz A. Glucocorticoids for croup. Cochrane Database Syst Rev 2004; 1: CD001955.

16. Bjornson C, Klassen T, Williamson J. A randomized trial of a single dose of oral dexamethasone for mild croup. N Engl J Med 2004; 351: 1306-13

17. Klassen T, Craig W, Moher D. Nebulized budesonide and oral dexamethasone for treatment of croup: a randomized controlled trial. JAMA 1998; 279: 1629-32

18. Amir L, Hubermann H, Halevi A, Mor M, Mimouni M, Waisman Y. Oral betamethasone versus intramuscular dexamethasone for the treatment of mild to moderate viral croup. Pediatr Emerg Care 2006; 22: 541-44 
19. Bjornson CL, Johnson DW. Croup. Lancet 2008; 371: 329-39

20. Sparrow A, Geelhoed G. Prednisolone versus dexamethasone in croup: a randomised equivalence trial. Arch Dis Child 2006; 91: 580-83 21. Kairys S, Marsh-Olmstead E, O'Connor G. Steroid treatment of laryngotracheitis: a meta-analysis of the evidence from randomized trials. Pediatr 1989; 83: 683-93

22. Russell KF, Liang Y, O'Gorman K, Johnson DW, Klassen TP. Cochrane Database Syst Rev. 2011 CD001955

23. Zhang L, Sanguebsche L. [The safety of nebulization with 3 to $5 \mathrm{ml}$ of adrenaline $(1: 1000)$ in children: an evidence based review.] J Pediatr (Rio J) 2005; 81: 193-97

24. Adair J, Ring W, Jordan W, Elwyn R. Ten-year experience with IPPB in the treatment of acute laryngotracheobronchitis. Anesth Analg 1971; 50: $649-55$

25. Kristjansson S, Berg-Kelly K, Winso E. Inhalation of racemic adrenaline in the treatment of mild and moderately severe croup: clinical symptom score and oxygen saturation measurements for evaluation of treatment effects. Acta Paediatr 1994; 83: 1156-60

26. Bjornson C, Russell KF, Vandermeer B, Durec T, Klassen TP, Johnson DW Nebulized Epinephrine for croup in children (Review). Cochrane Database Syst Rev. 2011 Feb 16;(2):CD00661

Los autores declaran no presentar conflicto de intereses. 